African Crop Science Journal by African Crop Science Society is licensed under a Creative Commons Attribution 3.0 Uganda License. Based on a work at www.ajol.info/ and www.bioline.org.br/cs DOI: http://dx.doi.org/10.4314/acsj.v23i4.6

\title{
COMPARAISON DE DEUX TECHNIQUES DE REPLANTATION CACAOYẼRE SUR ANTÉCÉDENTS CULTURAUX NON-FORESTIERS EN CÔTE D'IVOIRE
}

\author{
A.A. ASSIRI ${ }^{1}$, A. KONAN ${ }^{1}$, K.F. N'GUESSAN ${ }^{1}$, B.I. KÉBÉ ${ }^{2}$, K.E. KASSIN ${ }^{3}$, J.Y. COULOUD ${ }^{4}$, \\ A.R. YAPO ${ }^{5}$, G.R. YORO ${ }^{3}$ et A. YAO-KOUAMÉ ${ }^{6}$ \\ ${ }^{1}$ Centre National de Recherche Agronomique (CNRA), BP 808 Divo, Côte d'Ivoire \\ ${ }^{2}$ CNRA, Direction Générale, 01 BP 1740 Abidjan 01, Côte d'Ivoire \\ ${ }^{3}$ CNRA, 01 BP 633 Bouaké 01, Côte d'Ivoire \\ ${ }^{4}$ World Cocoa Foundation (WCF), Abidjan, Côte d'Ivoire \\ ${ }^{5}$ Le Conseil du Café-Cacao, 04 BP 2576 Abidjan 04, Côte d'Ivoire \\ ${ }^{6}$ Université Félix Houphouët-Boigny, UFR STRM, 22 BP 801 Abidjan 22, Côte d'Ivoire \\ Auteur correspondant : alexis.assiri@yahoo.fr
}

(Received 24 April, 2015; accepted 12 November, 2015)

\section{RÉSUMÉ}

Le verger de cacaoyers de la Côte d'Ivoire, premier producteur mondial de cacao, est vieillissant et doit être régénéré pour assurer une production durable de cacao. Mais, les réserves de forêt utilisées autrefois pour l'extension des plantations sont épuisées. Les terres cultivables disponibles sont les jachères et les cacaoyères dégradées. Malheureusement, la plupart des tentatives de plantation sur ces antécédents culturaux dégradés échouent. En vue de proposer aux producteurs des itinéraires techniques adaptés, deux techniques de replantation ont été comparées sur 40 parcelles replantées dont 20 après des vieux cacaoyers âgés de plus de 30 ans avec moins de 700 arbres ha ${ }^{-1}$ et 20 autres après des jachères de durée inférieure à 10 ans. Ces parcelles ont été installées entre 2004 et 2006 dans 10 régions productrices de cacao. Les résultats ont montré que la replantation sous vieux cacaoyers est plus efficace. L'efficacité de cette technique est liée à la fertilité des sols relativement meilleure et au niveau d'enherbement plus faible sous les vieux cacaoyers par rapport aux jachères. Il est cependant nécessaire de bien gérer l'ombrage des vieux cacaoyers, en effectuant des éclaircies avant 6 et 24 mois après la mise en place des jeunes cacaoyers.

Mots Clés : Cacao, itinéraires techniques, jachère, régénération

\begin{abstract}
Cocoa farms of Côte d'Ivoire, the world's largest cocoa producer, are getting older and must be regenerated for sustainable cocoa production. However, forest reserves formerly used for the expansion of plantations are finished. Available cultivable land parcels are fallows with poor soil and degraded cocoa farms. Unfortunately, most attempts of replanting on these poor lands fail. In order to provide producers with appropriate replanting techniques, two replanting techniques were compared on a 40 replanted plots, including 20 plots after more than 30 years-old cocoa farms with less than 700 trees ha $^{-1}$ and 20 other plots after fallows of less than 10 years. These plots were installed between 2004 and 2006 in 10 cocoa producing areas. Replanting under the old cocoa trees is more effective. The effectiveness of this practice is related to the relatively better soil fertility and lower level of the weeds proliferation under the old cocoa trees as compared to the fallows. It is, however, necessary to reduce the shade of the old cocoa trees before 6 and 24 months after the establishment of the young cocoa trees.
\end{abstract}

Key Words: Cocoa, fallow, previous land, regeneration 


\section{INTRODUCTION}

La Côte d'Ivoire est le premier producteur mondial de cacao depuis 1978. En 2014-2015, sa production a été de 1741000 tonnes, soit près de $42 \%$ de l'offre mondiale (ICCO, 2015). Le cacao représente un produit agricole stratégique pour la Côte d'Ivoire. En effet, il joue un rôle primordial dans la prospérité économique et sociale du pays. Au plan social, la culture du cacaoyer (Theobroma cacao L.) occupe une population agricole de plus d'un million de planteurs et procure de nombreux emplois dans les secteurs secondaire et tertiaire (Anonyme, 1999). Il est estimé qu'environ trois millions de personnes vivent des revenus du cacao (Anonyme, 2003). Au plan économique, le cacao procure environ $30 \%$ des recettes d'exportations et contribue, à hauteur de $10 \%$, au Produit Intérieur Brut (PIB) (Anonyme, 1999).

La durabilité de la culture et la production de cacao sont cependant menacées par de nombreuses contraintes, notamment le vieillissement des vergers et les difficultés de leur régénération (Ruf, 2000 ; Ruf et Allangba, 2001 ; Assiri et al., 2012). Ces difficultés sont d'abord liées à la pénurie de forêt, antécédent cultural idéal (Ruf, 1995) recherché par les producteurs pour l'établissement des plantations de cacaoyers. Face à l'épuisement des réserves forestières du pays qui sont passées de 16 millions d'hectares en 1960 à moins de 2,5 millions d'hectares dans les années 2000 (FAO, 2003), cet antécédent cultural est devenu de plus en plus rare, et a entrainé le ralentissement, voire l'arrêt des dynamiques d'extension cacaoyère depuis le début des années 1980 (Losch, 1994).

Par conséquent, le système traditionnel de culture extensive et itinérante du cacaoyer qui avait permis, jusqu'à cette époque, d'assurer le renouvellement du verger avait atteint ses limites et ne pouvait donc plus être reproduit (Léonard et Oswald, 1996). Ne trouvant plus de forêt dans le domaine rural, de nombreux producteurs se sont infiltrés dans la plupart des aires protégées et ont converti d'importantes surfaces en terres agricoles. En 1999, la Société de développement des forêts (SODEFOR) avait dénombré près de 80500 clandestins, chefs d'exploitations et estimé à environ 630120 ha, la superficie des plantations créées dans les forêts classées (FAO, 2003).
La pénurie de forêt pose donc la problématique du maintien de la production cacaoyère, qui a essentiellement résulté de l'accroissement des superficies et du déplacement des « boucles du cacao », au détriment de la forêt. Les difficultés de régénération des vieux vergers de cacaoyers sont également liées à l'évolution défavorable du climat (Freud et al., 2000 ; Brou et al., 2003 et 2005 ; Kassin, 2009) qui rend certaines régions de plus en plus marginales pour la culture du cacaoyer. A ces difficultés, s'ajoute la baisse de la fertilité des sols et le vieillissement des producteurs eux-mêmes.

Leur force de travail a ainsi fortement diminué et leurs revenus sont réduits par la sénescence des cacaoyers (Petithuguenin, 1995) dont la régénération exige cependant un nouvel investissement physique et financier important (Ruf, 2000). Face à toutes ces difficultés, la plupart des producteurs abandonnent leurs vieux vergers de cacaoyers ou les reconvertissent en plantations d'autres cultures pérennes, notamment l'hévéa et le palmier à huile (Naï Naï et al., 2000).

Dans ce contexte, pour contribuer à une production durable de cacao en Côte d'Ivoire, il importe de proposer aux producteurs des techniques adaptées pour l'installation des cacaoyères sur des antécédents culturaux nonforestiers. Des études récentes ont montré qu'à l'échelle des exploitations agricoles, les vieux vergers de cacaoyers et les jachères constituent les réserves foncières les plus importantes (Assiri et al., 2009). En moyenne, ils représentent $69 \%$ des replantations (Assiri, 2010). Ces deux antécédents culturaux n'offrent pas cependant les mêmes aptitudes que la forêt (Ruf, 2000 ; Clough et al., 2009), en raison de la dégradation de leurs sols, de l'assèchement du climat, ainsi que d'une pression plus forte des parasites et des mauvaises herbes comparativement à la forêt (Petithuguenin, 1995 ; Ruf, 2000). Dès lors, l'itinéraire technique utilisé lors de l'installation des cacaoyères sur défriches forestières ne peut plus convenir à la replantation sur ces antécédents culturaux (Jarrige et Ruf, 1990). Les échecs de nombreuses tentatives de replantation (Ruf et Allangba, 2001) confirment cette impossibilité de reproduire sur ces antécédents 
culturaux les techniques traditionnelles de culture du cacaoyer après la forêt.

Une étude a donc été entreprise entre 2004 et 2010, en vue d'évaluer l'effet de ces deux principaux antécédents culturaux et de leurs modes de préparation sur le développement des jeunes cacaoyers au cours de la replantation.

\section{MATÉRIEL ET MÉTHODES}

Zone d'étude. L'étude a été conduite dans 10 départements (Fig. 1) dont huit (Aboisso, Abengourou, Agnibilékrou, Daoukro, Bongouanou, Adzopé, Alépé et Agboville) à l'Est et au Sud-Est du pays, dans l'ancienne « boucle » de production de cacao, et deux (Divo et Tiassalé) au Sud. Ces départements représentent environ $35 \%$ du verger de cacao national. Ils sont caractérisés par des vergers vieillissants et des dynamiques de replantation (Assiri, 2007).

Le climat est de type subéquatorial, chaud et humide. La pluviométrie suit un régime bimodal, avec des hauteurs moyennes de pluie variant de 1200 à $1400 \mathrm{~mm} \mathrm{an}^{-1}$. Les températures moyennes annuelles sont comprises entre 24 et $32^{\circ} \mathrm{C}$ (Kassin et al., 2008). La durée d'insolation (plus de 1700 heures $a^{-1}$ ) et l'humidité relative (plus de $80 \%$ ) sont élevées durant toute l'année (Brou, 2005). La végétation naturelle était la forêt dense humide et couvrait près de 16 millions d'hectares dans les années 1960. Elle a été surexploitée et il n'en reste plus que des reliques estimées à moins de 2,5 millions d'hectares (FAO, 2003). Les sols de la zone d'étude sont en majorité des Ferralsols (FAO, 2006 ; 2009). Le pH est proche de 6,0 dans la couche $0-20 \mathrm{~cm}$ de profondeur. La fertilité chimique est faible à moyenne, avec des déficiences en phosphore et en potassium, au regard des exigences du cacaoyer (Assiri, 2010).

Matériel. Le matériel biologique utilisé était composé de :

(i) deux antécédents culturaux non-forestiers : il s'agit de jachères à Chromolaena odorata (L.) King \& Robinson (Asteraceae) de durée inférieure à 10 ans et de vieilles cacaoyères paysannes de plus de 30 ans, avec environ 700 arbres ha ${ }^{-1}$ et des rendements moyens inférieurs à $200 \mathrm{~kg} \mathrm{ha}^{-1} \mathrm{an}^{-1}$; (ii) jeunes plants hybrides de cacaoyers âgés de six à sept mois ; ils ont été utilisés pour la replantation selon un dispositif en rectangle ( $3 \mathrm{~m} \times 2,5 \mathrm{~m}$ ), soit une densité de 1333 arbres $\mathrm{ha}^{-1}$

(iii) bananiers plantains sous forme de rejets et Gliricidia sepium (Jacq.) Walp (Fabaceae) sous forme de chicots d'au moins $1,5 \mathrm{~m}$ de haut ; ils ont été utilisés comme plantes d'ombrage temporaire des jeunes cacaoyers, dans le cas des antécédents jachères.

Dispositif expérimental. Le dispositif expérimental était constitué par un réseau de 40 cacaoyères replantées en milieu paysan sur au moins 0,25 ha chacune. Vingt (20) ont été installées après des vieux cacaoyers (technique de replantation T1) et 20 autres après des jachères (T2). Chaque parcelle a été considérée comme une répétition.

Avant la mise en place des jeunes cacaoyers qui a eu lieu en mai-juin, chaque antécédent cultural a fait l'objet d'un mode de préparation spécifique (Tableau 1). Pour les vieux cacaoyers (T1), aucun ombrage temporaire n'a été installé. Par contre, pour les jachères (T2), des bananiers plantains et G. sepium ont été plantés, en les alternant aux écartements de $3 \mathrm{~m}$ x $5 \mathrm{~m}$, soit 666 pieds ha ${ }^{-1}$ pour chaque plante (Fig. 2). Ils ont été installés la même année que les cacaoyers. Après la mise en place des cacaoyers, la conduite des parcelles a consisté en des entretiens classiques et en des reglages de l'ombrage des vieux cacaoyers (T1) et de G. sepium (T2), à la demande, suivant le développement des jeunes cacaoyers.

Paramètres mesurés. Les observations ont été effectuées sur les sols et sur les jeunes cacaoyers replantés en fonction des antécédents culturaux. Sur les sols, les observations avaient pour but d'évaluer la fertilité chimique initiale. Ainsi, un échantillon composite de sol a été prélevé par site, soit un total de 40 échantillons. Un échantillon composite est issu d'un mélange d'au moins 20 prélèvements par site, dans la couche 0-20 cm de profondeur comme dans le cas de la méthode du « diagnostic-sol » (Snoeck, 2006). Les analyses ont été faites selon les méthodes et les procédures standards décrites par Van Reeuwijk (2006). Les paramètres déterminés sont 
TABLEAU 1. Modes de préparation des terrains en fonction des antécédents culturaux

\begin{tabular}{|c|c|c|}
\hline \multirow[t]{2}{*}{ Succession des travaux de préparation du terrain et d'entretien des parcelles replantées } & \multicolumn{2}{|c|}{ Antécédents culturaux } \\
\hline & Vieux cacaoyers & Jachères \\
\hline \multicolumn{3}{|l|}{ Préparation des terrains : } \\
\hline Fauchage & $x$ & $x$ \\
\hline Brûlis ou traitement herbicide & & $\mathrm{x}$ \\
\hline Piquetage $(3 \mathrm{~m} \times 2,5 \mathrm{~m})$ & & $x$ \\
\hline Piquetage $(3 \mathrm{~m} \times 2,5 \mathrm{~m})$ sous les vieux cacaoyers & $\mathrm{x}$ & \\
\hline $\begin{array}{l}\text { Ouverture des nouvelles lignes de plantation (abattage et taille des vieux cacaoyers } \\
\text { situés à moins de } 50 \mathrm{~cm} \text { ) }\end{array}$ & $\mathrm{x}$ & \\
\hline Mise en place des plantes d'ombrage temporaire (bananiers, Gliricidia sepium) & & $x$ \\
\hline Trouaison $(40 \mathrm{~cm} \times 40 \mathrm{~cm} \times 40 \mathrm{~cm})$ & $\mathrm{x}$ & $x$ \\
\hline Mise en place des jeunes cacaoyers & $x$ & $x$ \\
\hline \multicolumn{3}{|l|}{ Entretien des parcelles replantées : } \\
\hline Désherbage, égourmandage, taille de formation, traitements insecticides & $x$ & $x$ \\
\hline Réglages de l'ombrage des vieux cacaoyers (à la demande) & $x$ & \\
\hline Réglage de l'ombrage du G. sepium (éclaircie à la demande) & & $x$ \\
\hline
\end{tabular}

le $\mathrm{pH}$ eau, les bases échangeables, la capacité d'échange cationique (CEC), le carbone organique (C), l'azote total ( $\mathrm{N}$ total), le phosphore total ( $\mathrm{P}$ total) et assimilable ( $\mathrm{P}$ ass.). De ces paramètres, ont été déduits :

(i) la matière organique (MO), par la relation : $\mathrm{MO}$ $=1,724 \times \mathrm{C}$;

(ii) le rapport carbone sur azote $(\mathrm{C} / \mathrm{N})$;

(iii) la somme des bases échangeables (S) ;

(iv) le taux de saturation du complexe adsorbant (V \%) par la relation : (S/CEC) x 100 ; et

(v) les équilibres anioniques et cationiques pour une nutrition minérale et une production optimale des cacaoyers (Snoeck, 2006).

Sur les jeunes cacaoyers, les observations ont porté sur la mortalité, la croissance, la formation de couronne et la précocité de production. Ainsi, le nombre de plants morts et ceux qui ont couronné ont été déterminés à 6,12 et 20 mois après la mise en place. Aux mêmes dates, la hauteur totale et le diamètre au collet des plants à $10 \mathrm{~cm}$ au-dessus de la surface du sol ont été mesurés, respectivement à l'aide d'une règle graduée de $2 \mathrm{~m}$ de long et d'un pied à coulisse. L'âge d'entrée en floraison des jeunes cacaoyers a été également noté afin d'apprécier leur précocité de production (Lachenaud, 1987). En vue d'apprécier le niveau d'enherbement des parcelles replantées, la biomasse fraîche des mauvaises herbes a été déterminée. Les observations ont été faites uniquement dans les parcelles de Divo, au cours de la première année de plantation. Trois mesures ont été effectuées par parcelle, à l'aide d'un gabarit de $1 \mathrm{~m}^{2} \mathrm{de}$ surface, à différentes dates, avant chaque désherbage.

Analyses des données. Les analyses statistiques ont été faites avec le logiciel XLSTAT Version 2009.4.02. Il s'est agi de statistiques descriptives et d'analyses de variance (ANOVA). Le test de Bonferroni au seuil de $5 \%$ a été utilisé pour la comparaison des moyennes.

\section{RÉSULTATS}

Fertilité chimique initiale des sols en fonction des antécédents culturaux. Les résultats sont consignés dans le Tableau 2. Ils concernent les caractéristiques de la matière organique, la réaction du sol et le complexe adsorbant, puis les équilibres chimiques.

\section{Caractéristiques de la matière organique des} sols. Les teneurs moyennes ont été relativement plus élevées sous les vieux cacaoyers que sous les jachères, sauf pour le rapport $\mathrm{C} / \mathrm{N}$. Cependant, 


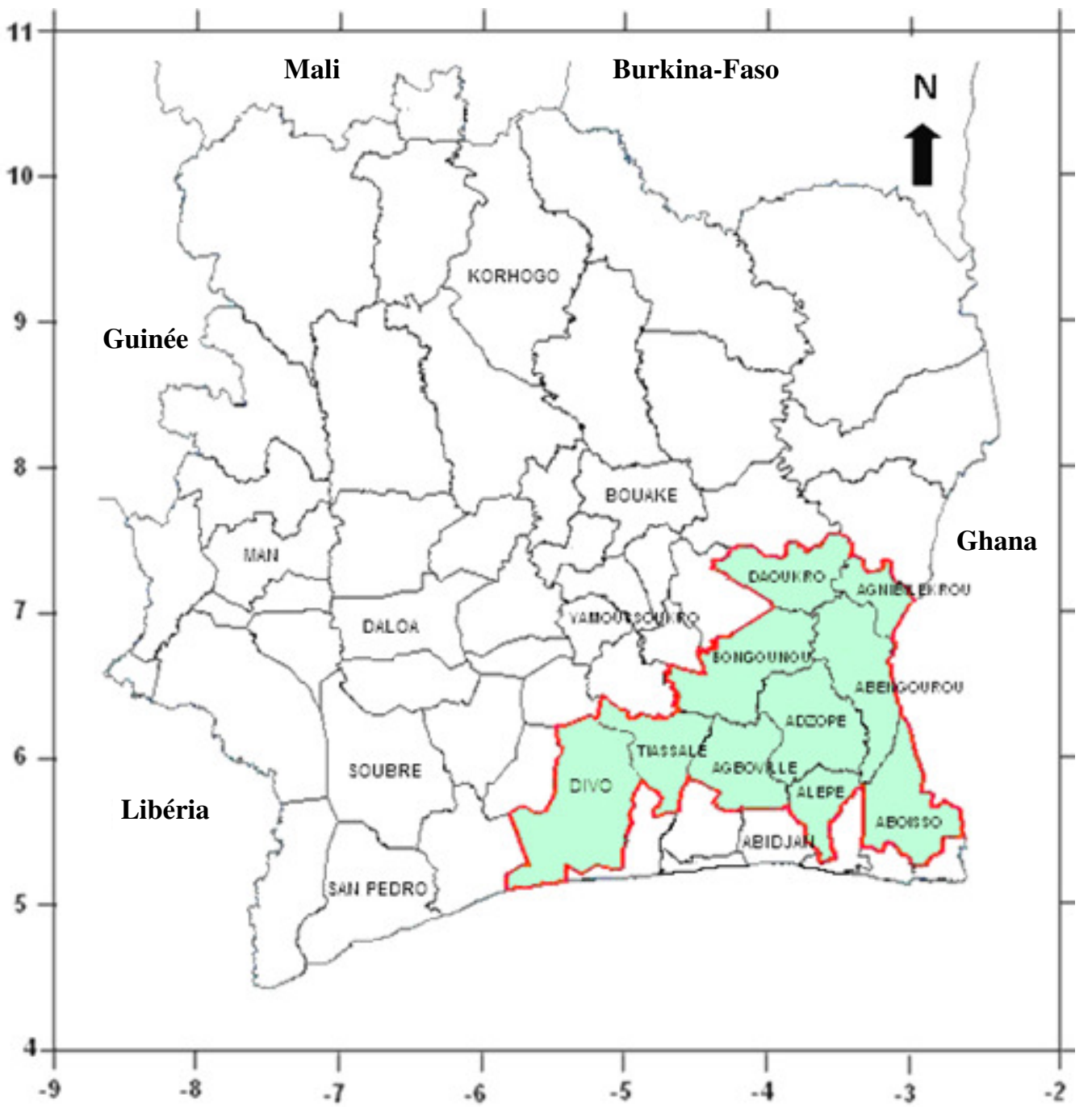

Légende : $\square$ : Zone d'Étude

Figure 1. Localisation de la zone d'étude et des 10 départements couvrant le réseau de 40 parcelles de replantation cacaoyère

comparativement à la valeur optimale $\left(35 \mathrm{~g} \mathrm{~kg}^{-1}\right.$ de matière organique), les teneurs moyennes de la couche $0-20 \mathrm{~cm}$ sous les vieux cacaoyers $(24,1$ $\left.\mathrm{g} \mathrm{kg}^{-1}\right)$ et les jachères $\left(17,8 \mathrm{~g} \mathrm{~kg}^{-1}\right)$ se sont révélées faibles (Tableau 2). Seulement 23,5\% des échantillons sous vieux cacaoyers ont présenté des teneurs proches de l'optimum. En ce qui concerne le rapport $\mathrm{C} / \mathrm{N}$, les moyennes observées ont été sensiblement plus satisfaisantes sous les jachères $(10,93)$ que sous les vieux cacaoyers $(8,77)$.
Réaction du sol et complexe adsorbant. Les $\mathrm{pH}$ eau des sols sous les vieux cacaoyers $(6,12)$ et les jachères $(6,07)$ étaient comparables et se situaient dans l'intervalle des valeurs (5 à 7) favorables au cacaoyer (Tableau 2). Au niveau du complexe adsorbant, les sols sous vieux cacaoyers ont présenté des teneurs en bases échangeables $\left(\mathrm{Ca}^{++}, \mathrm{Mg}^{++}\right.$et $\left.\mathrm{K}^{+}\right)$sensiblement plus élevées que celles déterminées sous jachères. La capacité d'échange cationique (CEC) a varié de $10,02 \mathrm{cmol} \mathrm{kg}^{-1}$ sous les jachères à 9,65 


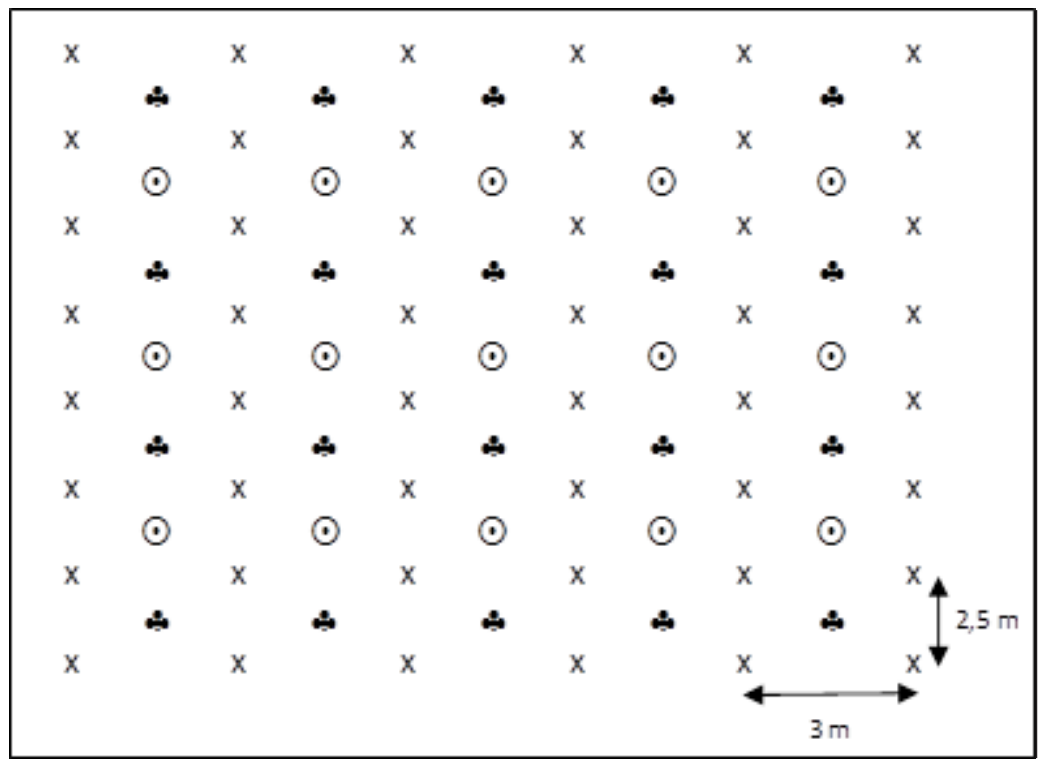

Légende: $\mathrm{X}$ : Cacaoyer $(3 \mathrm{~m}$ x 2,5 m)

$\odot:$ Bananier plantain $(3 \mathrm{~m} \times 5 \mathrm{~m})$

: Gliricidia sepium (3 m x $5 \mathrm{~m})$

Figure 2. Schéma du dispositif d'installation de l'ombrage mixte de bananiers plantains et Gliricidia sepium dans les replantations après jachères (T2).

cmol kg-1 sous les vieux cacaoyers. Ces valeurs sont plus faibles que l'optimum (au moins $12 \mathrm{cmol}$ $\mathrm{kg}^{-1}$ ) qui n'a été atteint que seulement dans 16,67 $\%$ des sols des jachères et $29,41 \%$ pour les vieux vergers de cacaoyers. Quant au taux de saturation en bases $(\mathrm{V})$, il a varié de $41,66 \%$ sous jachères à $55,11 \%$ sous les vieux cacaoyers. L'optimum (plus de $60 \%$ ) a concerné une proportion de 47,06 $\%$ des sols des cacaoyères, contre $16,67 \%$ pour les jachères. Concernant le phosphore, les teneurs moyennes des sols pour les formes totale $(210,67$ à $335,94 \mathrm{mg} \mathrm{kg}^{-1}$ ) et assimilable de cet élément $\left(25,83\right.$ à $\left.63,18 \mathrm{mg} \mathrm{kg}^{-1}\right)$ étaient très faibles dans tous les sols, quel que soit l'antécédent cultural. Seulement 23,5\% des sols sous vieux cacaoyers en était bien pourvus, avec plus de $100 \mathrm{mg} \mathrm{kg}^{-1}$ de phosphore assimilable.

Equilibres chimiques. Les valeurs de six équilibres chimiques ont été déterminées (Tableau 2). Pour le rapport azote total ( $N$ tot.) sur phosphore total (P tot.), les valeurs ont varié de
5,92 dans les sols sous jachères, à 8,09 sous les cacaoyères, soit environ 3 à 4 fois plus élevé que l'optimum (1,5 à 2). Concernant les cations, leurs proportions par rapport à la somme des bases échangeables (S) ont varié de 69,49 à 74,54\% pour le calcium $\left(\mathrm{Ca}^{++}\right)$et de 22,58 à $24,13 \%$ pour le magnésium $\left(\mathrm{Mg}^{++}\right)$. Ces valeurs étaient proches des taux recherchés $\left(68 \%\right.$ pour $\mathrm{Ca}^{++}$et $24 \%$ pour et $\mathrm{Mg}^{++}$). Comparés à l'optimum de $8 \%$, les taux moyens (2,88 à $6,38 \%)$ concernant le potassium $\left(\mathrm{K}^{+}\right)$étaient par contre faibles sur les deux antécédents culturaux. Pour le rapport $\mathrm{Ca}^{++} / \mathrm{Mg}^{++}$, les valeurs moyennes observées s'élevaient à 4,59 dans les sols des vieilles cacaoyères et à 3,84 sous les jachères. Elles étaient proches de l'optimum (1 à 4).Concernant le rapport $\mathrm{Mg}^{++}$/ $\mathrm{K}^{+}$, les valeurs moyennes ont varié de 8,16 dans les sols sous vieux cacaoyers à 11,32 sous les jachères, soit environ 2 à 3 fois plus élevées que l'optimum (3,5 à 4). Pour le rapport de la somme du calcium et du magnésium sur le potassium $\left(\mathrm{Ca}^{++}+\mathrm{Mg}^{++} / \mathrm{K}^{+}\right)$, les moyennes étaient 
TABLEAU 2. Caractéristiques de la fertilité chimique initiale de la couche $0-20 \mathrm{~cm}$ des sols en fonction des antécédents culturaux utilisés pour la replantation cacaoyère

\begin{tabular}{|c|c|c|c|c|c|}
\hline \multicolumn{2}{|c|}{ Caractéristiques de la fertilité chimique des sols } & \multicolumn{2}{|c|}{ Antécédents vieux cacaoyers(plus de 20 ans) } & \multicolumn{2}{|c|}{ Antécédents jachères(moins de 10 ans) } \\
\hline Paramètres & $\begin{array}{l}\text { Valeurs optimales } \\
\text { pour le cacaoyer }\end{array}$ & Moyennes & $\begin{array}{l}\text { Échantillons ayant des } \\
\text { valeurs optimales (\%) }\end{array}$ & Moyennes & $\begin{array}{l}\text { Échantillons ayant des } \\
\text { valeurs optimales (\%) }\end{array}$ \\
\hline \multicolumn{6}{|c|}{ Matière organique $\left(\mathrm{g} \mathrm{kg}^{-1} \mathrm{de}\right.$ sol) : } \\
\hline C & - & $14,0 \pm 3,9$ & - & $10,3 \pm 2,6$ & - \\
\hline $\mathrm{N}$ tot. & - & $1,7 \pm 0,4$ & - & $1,1 \pm 0,4$ & - \\
\hline MO & ${ }^{3} 35$ & $24,1 \pm 6,7$ & 23,5 & $17,8 \pm 4,6$ & 0,0 \\
\hline $\mathrm{C} / \mathrm{N}$ & 9 à 12 & $8,77 \pm 1,03$ & 35,3 & $10,93 \pm 3,60$ & 50,0 \\
\hline \multicolumn{6}{|c|}{ pH et complexe adsorbant $\left(\mathrm{cmol} \mathrm{kg}^{-1}\right)$ : } \\
\hline pH eau & 5 à 7 & $6,12 \pm 0,46$ & 64,7 & $6,07 \pm 0,36$ & 100,0 \\
\hline P tot. & ${ }^{3} 560$ & $335,94 \pm 88,51$ & 5,9 & $210,67 \pm 82,96$ & 0,0 \\
\hline$P$ ass. & 3100 & $63,18 \pm 27,87$ & 23,5 & $25,83 \pm 15,33$ & 0,0 \\
\hline CEC & ${ }^{3} 12$ & $9,65 \pm 1,32$ & 29,4 & $10,02 \pm 1,41$ & 16,7 \\
\hline $\mathrm{Ca}++$ & - & $4,30 \pm 1,67$ & $\begin{array}{r}2 v, 7 \\
-\end{array}$ & $3,09 \pm 1,27$ & - \\
\hline $\mathrm{Mg}++$ & - & $0,86 \pm 0,24$ & - & $0,83 \pm 0,15$ & - \\
\hline $\mathrm{K}+$ & - & $0,35 \pm 0,17$ & - & $0,12 \pm 0,07$ & - \\
\hline $\mathrm{S}$ & - & $5,51 \pm 1,98$ & - & $4,04 \pm 1,34$ & - \\
\hline$V(\%)$ & ${ }^{3} 60$ & $55,11 \pm 16,94$ & 47,1 & $41,66 \pm 16,60$ & 16,7 \\
\hline \multicolumn{6}{|c|}{ Equilibres chimiques : } \\
\hline $\mathrm{N}$ tot./P tot. & 1,5 à 2 & $8,09 \pm 4,21$ & & $5,92 \pm 2,84$ & \\
\hline $\mathrm{K}+-\mathrm{Ca}^{++}-\mathrm{Mg}^{++}(\% \mathrm{~S})$ & $8-68-24$ & $6,4-69,5-24,1$ & & $2,9-74,5-22,6$ & \\
\hline $\mathrm{Ca}^{++} / \mathrm{Mg}^{++}$ & 1 à 4 & $\begin{array}{r}4,58 \pm 1,58\end{array}$ & & $\begin{array}{r}3,84 \pm 1,73 \\
3,84,-2<, 0\end{array}$ & \\
\hline $\mathrm{Mg}^{++} / \mathrm{K}^{+}$ & 3,5 à 4 & $8,16 \pm 4,43$ & & $11,32 \pm 5,75$ & \\
\hline$\left(\mathrm{Ca}^{++}+\mathrm{Mg}^{++}\right) / \mathrm{K}^{+}$ & $\begin{array}{r}0,0 \mathrm{~d} 4 \\
25\end{array}$ & $33,50 \pm 19,12$ & & $49,82 \pm 24,48$ & \\
\hline$(\mathrm{S}+6,15) / \mathrm{N}$ tot. & 8,9 & $7,82 \pm 1,05$ & & $11,39 \pm 4,31$ & \\
\hline
\end{tabular}

$\mathrm{C}:$ carbone organique $; \mathrm{N}$ tot. : azote total ; $\mathrm{MO}:$ matière organique $; \mathrm{C} / \mathrm{N}$ : rapport carbone $(\mathrm{C})$ sur azote $(\mathrm{N}) ; \mathrm{P}$ tot. : Phosphore total ; P ass. : Phosphore assimilable ; $\mathrm{CEC}:$ capacité d'échange cationique ; $\mathrm{S}$ : somme des bases échangeables ; $\mathrm{V}$ : taux de saturation en bases du complexe adsorbant. 
comprises entre 33,50 sous les cacaoyers et 49,82 sous les jachères. Ces valeurs étaient plus élevées que l'optimum qui est proche de 25,0. Quant à l'équilibre entre les bases échangeables et l'azote $[(\mathrm{S}+6,15) / \mathrm{N}$ total $]$ dont l'optimum est de 8,9 , les valeurs calculées étaient comprises entre 7,82 sous les vieilles cacaoyères et 11,39 sous les jachères.

Effets des techniques de replantation sur le développement des cacaoyers

Mortalité des jeunes cacaoyers. Les taux de mortalité enregistrés chez les jeunes cacaoyers entre 6 et 24 mois après la mise en place ont varié de 3,1 à 13,1\% (Fig. 3). À chaque stade de développement des cacaoyers, les valeurs observées dans les replantations après jachères (T2) étaient sensiblement plus élevées que sous les vieux cacaoyers (T1). Les moyennes sont statistiquement différentes à partir de 12 mois $(\mathrm{P}$ $=0,04$ ) après la mise en place des parcelles.

\section{Croissance en hauteur et diamètre au collet des} cacaoyers. La hauteur totale des plants a varié de 76,5 à 196,9 cm entre 6 et 12 mois (Fig. 4 A). Dans la même période, les diamètres au collet sont passés de 1,1 à 4,0 cm (Fig. 4 B). Aucune différence significative n'a été observée entre les deux techniques de replantation, quel que soit l'âge des plants. Il est cependant noté que sous vieux cacaoyers, les plants ont présenté des hauteurs moyennes sensiblement plus élevées qu'après jachère, mais avec des diamètres aux collets moins grands. Ainsi, à tous les stades de développement, le rapport de la hauteur totale (H) sur le diamètre au collet (D) des jeunes cacaoyers (Fig. 4 C) a été plus élevé sous les vieux cacaoyers $(65,5$ à 78,3$)$ que sur les jachères $(40,4$ à 54,0). Les différences ont été significatives $(\mathrm{P}<0,05)$ à 6 et 24 mois après la mise en place des parcelles.

Niveau d'enherbement des parcelles. La masse fraîche des mauvaises herbes sous vieux cacaoyers (T1) était comprise entre 1,0 et $1,89 \mathrm{t}$ $\mathrm{ha}^{-1}$, contre 6,11 à $6,78 \mathrm{t} \mathrm{ha}^{-1}$ sur les antécédents jachères (Tableau 3). A toutes les périodes considérées, les moyennes obtenues ont été trois à six fois plus élevées dans les replantations après jachères (T2) que sous les vieux cacaoyers (T1).

Précocité de production. À 24 mois après la mise en place, le taux de couronnement a été de 90,4\% chez les jeunes cacaoyers replantés sous les vieux (T1), contre 70,7 \% chez ceux installés après les

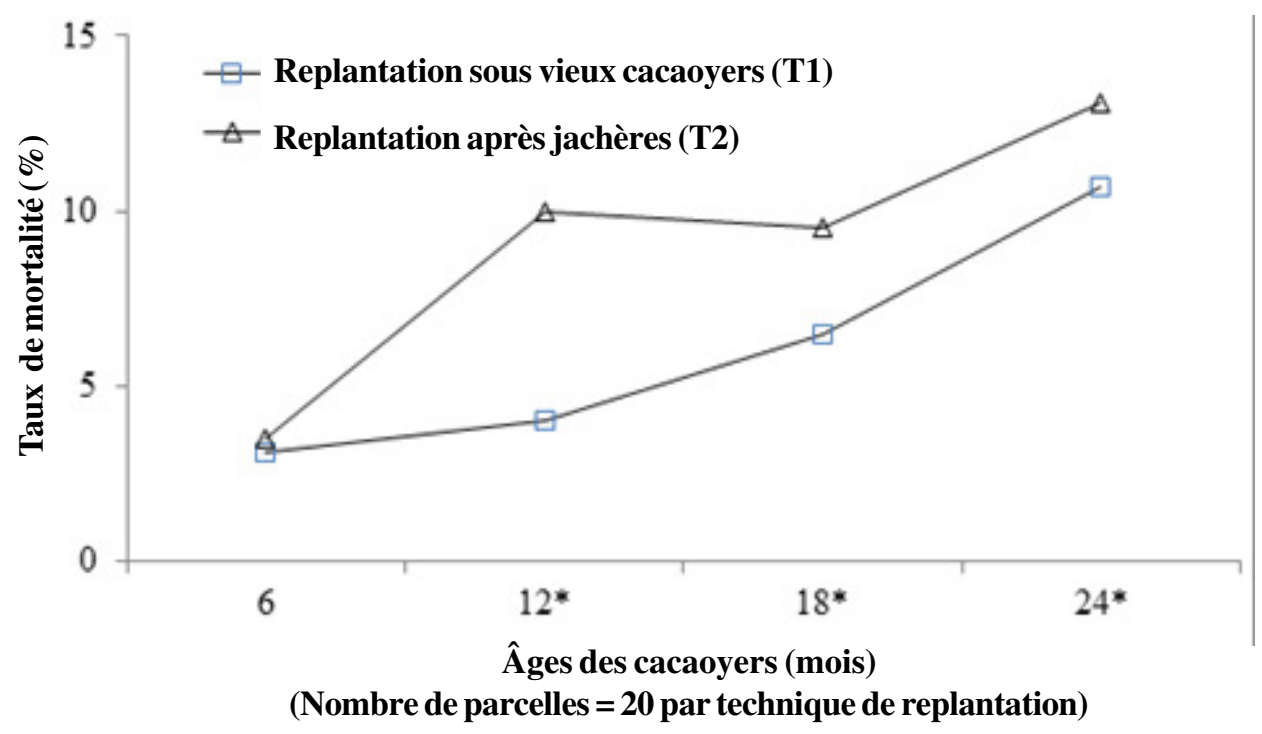

* : Moyennes statistiquement différentes (test de Bonferroni, $\mathrm{P}=5 \%$ )

Figure 3. Evolution des taux de mortalité des cacaoyers dans les replantations sous vieux cacaoyers et après jachère. 


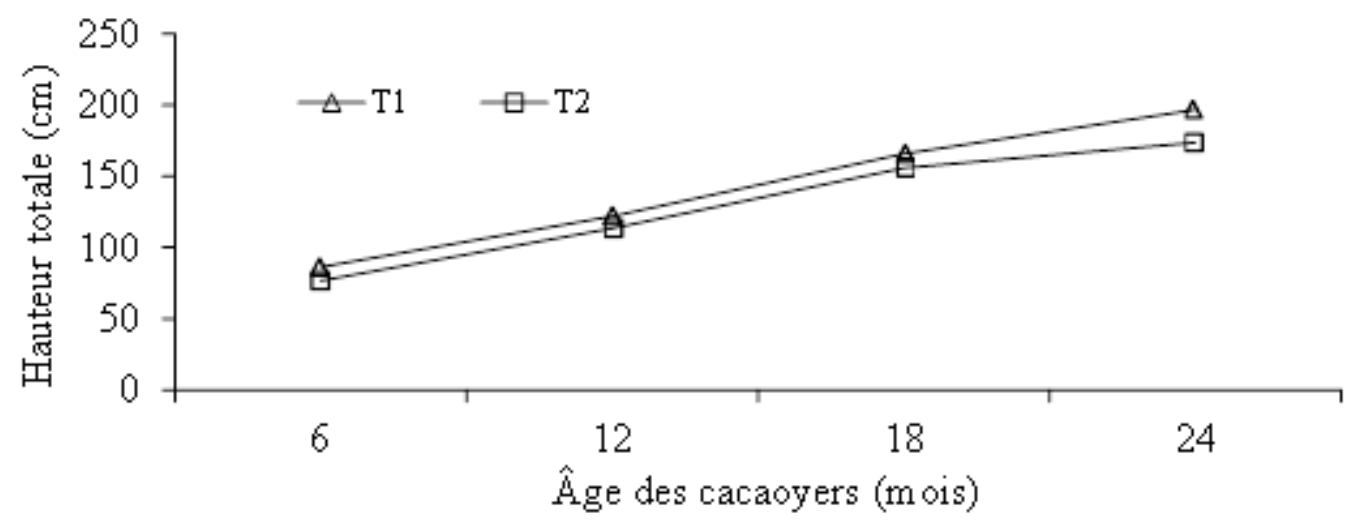

A : Hauteur

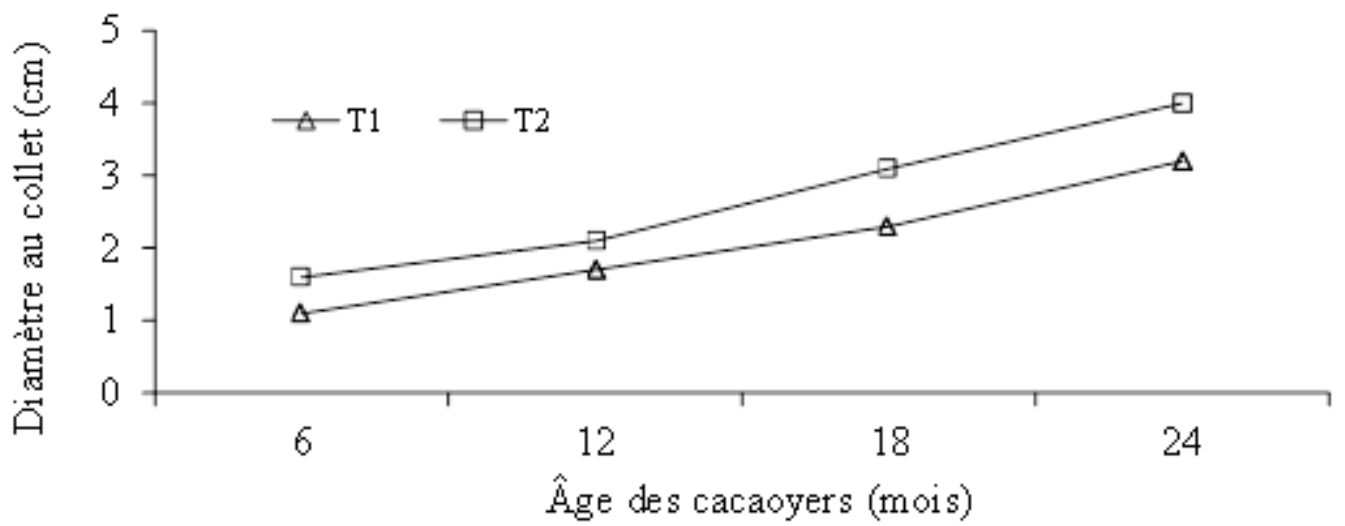

$B$ : Diametre au collet

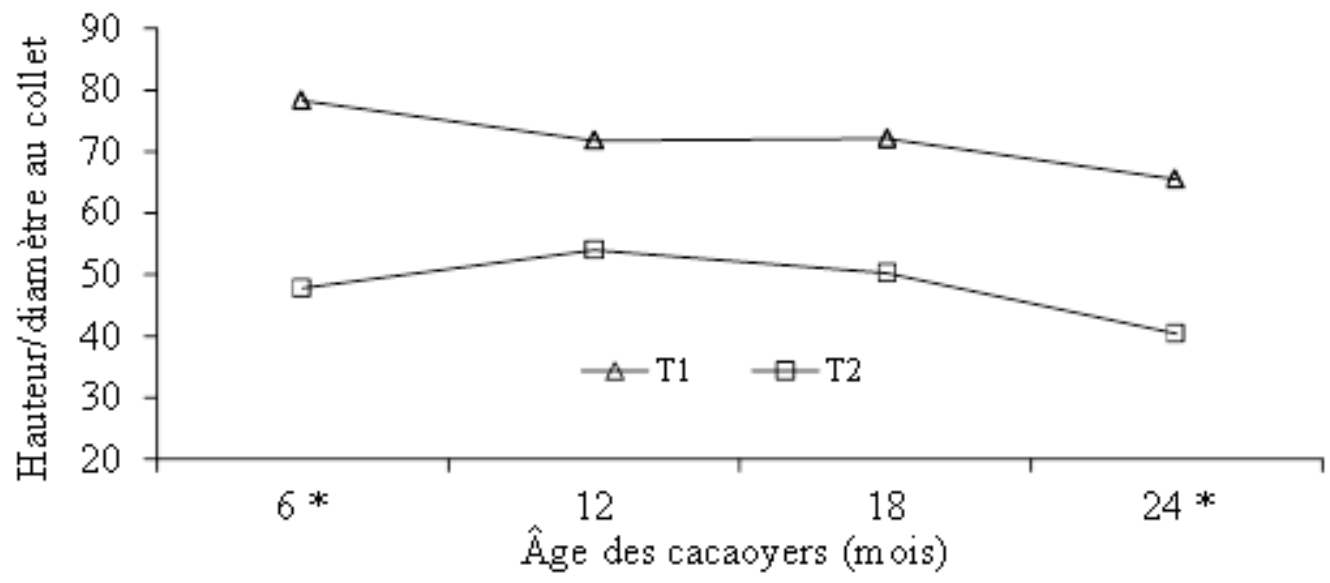

$\mathrm{C}:$ Hauteur / diamètre au collet

$\mathrm{T} 1$ : Replantation sous vieux cacaoyers ; T2 : Replantation après jachère; * : différence significative à $\mathrm{P}<5 \%$ (test de Bonferroni)

Figure 4 (A, B et C). Évolution des paramètres de croissance des jeunes cacaoyers entre 6 et 24 mois en fonction de la technique de replantation. 
TABLEAU 3. Biomasse des mauvaises herbes dans les replantations de Divo

\begin{tabular}{lccc}
\hline Biomasse des mauvaises herbes(tonne de matière fraîche ha- ${ }^{-1}$ ) & \multicolumn{2}{c}{ Âge des jeunes cacaoyers (mois) après la mise en place } \\
\cline { 2 - 4 } & 5 & 9 & 12 \\
\hline Replantation sous vieux cacaoyers (T1) & $1,44 \mathrm{~b}$ & $1,00 \mathrm{~b}$ & $1,89 \mathrm{~b}$ \\
Replantation après jachère (T2) & $6,78 \mathrm{a}$ & $6,11 \mathrm{a}$ & $6,72 \mathrm{a}$ \\
Moyennes de T2 / T1 & 4,708 & 6,110 & 3,556 \\
Différence entre les moyennes & & & $* * *$ \\
\hline
\end{tabular}

$-a, b$ : dans une même colonne, les moyennes n'ayant pas les mêmes lettres sont statistiquement différentes (test de Bonferroni au seuil de $5 \%) ;-^{* *},{ }^{* * *}$ : différence significative entre les moyennes, respectivement à $P$ inférieure à $1 \%$ et $0,1 \%$

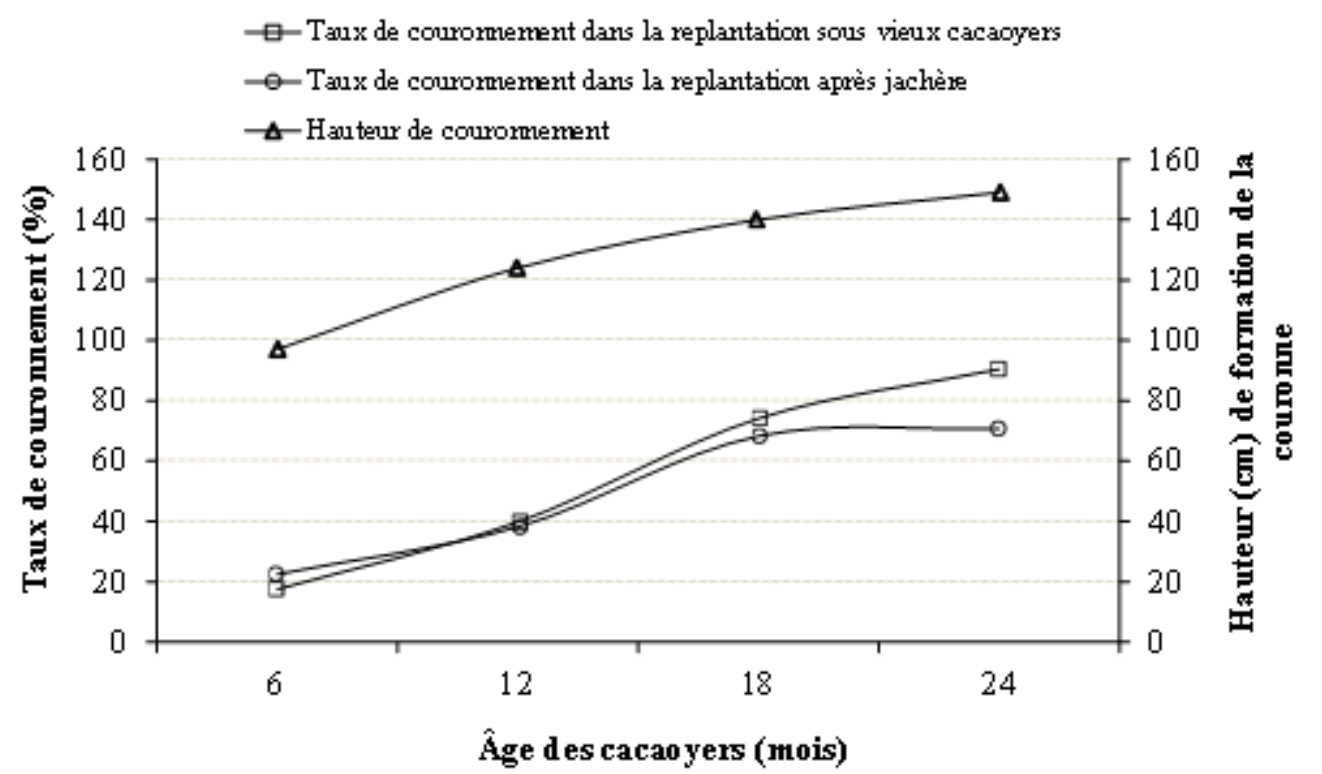

Figure 5. Évolution du taux et de la hauteur de couronnement des cacaoyers.

TABLEAU 4. Âge (mois) d'entrée en production des jeunes cacaoyers en fonction de la technique de replantation

\begin{tabular}{lcccc}
\hline Technique de replantation & Âge minimum & Âge maximum & Âge moyen & $\begin{array}{c}\text { Coefficient de } \\
\text { variation (\%) }\end{array}$ \\
\hline Replantation sous vieux cacaoyers & 18 & 36 & 26,7 & 25,6 \\
Replantation après jachère & 20 & 27 & 24,0 & 9,0 \\
\hline
\end{tabular}

jachères (Fig. 5). Les couronnes sont apparues à des hauteurs moyennes comprises entre 97 et $149 \mathrm{~cm} \mathrm{du}$ sol. Les taux de couronnement observés entre 6 et 24 mois étaient statistiquement comparables entre les deux techniques de replantation.
Les cacaoyers couronnés sont entrés en production entre 18 et 36 mois après la mise en place (Tableau 4). Les moyennes d'âge dans les replantations sous vieux cacaoyers (26,7 mois) et après jachères (24,0 mois) ont été statistiquement comparables. Toutefois, elles ont 
présenté une plus grande variabilité sous vieux cacaoyers (coefficient de variation $=25,6 \%$ ) qu'après les jachères (coefficient de variation = 9,0\%) (Tableau 4).

\section{DISCUSSION}

Les deux techniques de replantation étudiées ont été comparées en évaluant la mortalité, la croissance et la précocité de production des jeunes cacaoyers. Concernant la mortalité, les résultats obtenus ont montré qu'elle a été plus faible (3 à $12 \%$ ) sous vieux cacaoyers (T1) qu'après les antécédents jachères (T2). Des résultats similaires avaient été obtenus au Togo, avec des taux de mortalité de 3 à $15 \%$ sous les vieux cacaoyers, contre 30 à $50 \%$ en replantation totale (Vaast, 1987 ; Bertrand et Jadin, 1992). La fertilité des sols relativement meilleure et le faible niveau d'enherbement des parcelles après vieux cacaoyers (3 à 6 fois moins) par rapport aux jachères pourraient expliquer ces résultats. Les antécédents vieux cacaoyers auraient donc offert des conditions plus favorables pour l'installation et le développement des jeunes cacaoyers. En effet, compte tenu du faible enherbement des parcelles, il y aurait eu peu de compétition entre les jeunes cacaoyers et les mauvaises herbes pour l'eau du sol, les éléments minéraux et la lumière. Au contraire, sur antécédents jachères de courte durée, il a été démontré que les mauvaises herbes prolifèrent plus (Ruf, 2000 ; Gnahoua, 2004) et prélèvent dans le sol, des quantités d'azote, de phosphore et de potassium plus élevées que les cacaoyers (Shenk, 1971). Le contrôle régulier des mauvaises herbes est donc indispensable pour l'établissement des jeunes cacaoyers. Il est recommandé d'effectuer 4 à 6 désherbages par an pendant les deux à trois premières années (Oppong et al., 2006 ; Kébé et al., 2009). Le désherbage constitue cependant un goulot d'étranglement en milieu paysan. En effet, selon Oppong et al. (2003), il est l'opération culturale la plus onéreuse, représentant environ le quart du coût total de la main-d'œuvre.

Outre la bonne fertilité des sols et le faible niveau d'enherbement des parcelles, les jeunes cacaoyers auraient aussi bénéficié d'un ombrage plus favorable sous les vieux cacaoyers que sous le peuplement mixte de bananiers plantains et de
G. sepium, utilisé pour l'ombrage temporaire après les antécédents jachères. Toutefois, une contrainte liée à la gestion des vieux cacaoyers a été décelée. En effet, les valeurs élevées des rapports des hauteurs sur les diamètres au collet (H/D) indiquent que les jeunes cacaoyers avaient « filé » (Besse, 1972 ; Lachenaud, 1987), c'est-àdire, qu'ils se sont plus développés en hauteur, à la recherche de la lumière pour la photosynthèse. En conséquence, dans certaines parcelles, ils sont apparus moins vigoureux que ceux installés après jachères. Ce type de développement serait dû à un ombrage excessif des vieux cacaoyers à la mise en place des parcelles. Des fractions insuffisantes de radiations lumineuses parvenaient probablement aux jeunes cacaoyers. Les différences significatives observées entre les deux techniques de replantation pour le rapport H/D suggèrent qu'il étaient nécessaire de diminuer progressivement l'ombrage des vieux cacaoyers, en réalisant une première éclaircie avant le $6^{\text {ème }}$ mois après la mise en place, puis, une autre avant 24 mois. À la première éclaircie, la densité des vieux cacaoyers (en moyenne 700 abres $\mathrm{ha}^{-1}$ ) pourrait être diminuée de moitié, c'està-dire ramenée entre 300 et 400 pieds ha ${ }^{-1}$ comme le suggèrent Aguilar et Jadin (1997). Ces observations rappellent la méthode « Turrialba » au Costa Rica et au Brésil, qui consiste à réaliser une taille forte des frondaisons des vieux cacaoyers avant la mise en place des jeunes cacaoyers (Wood and Lass, 1986). Toutefois, sur le terrain, l'abattage des vieux cacaoyers est souvent différé, à cause de l'amélioration de leur production, suite au meilleur entretien de la parcelle (réhabilitation) au cours de la replantation. Les planteurs ont donc tendance à les maintenir plus longtemps, en vue de continuer à les exploiter.

Pourtant, outre l'effet dépressif sur la croissance, l'excès d'ombrage des vieux cacaoyers semble empêcher les jeunes plants de bien produire, en raison probablement d'une concurrence pour l'eau, les éléments minéraux et la lumière, qui limiterait la photosynthèse et la floraison. Ces raisons pourraient expliquer le retard d'entrée en production observé chez les jeunes cacaoyers dans les replantations sous les vieux vergers. Ce retard avait atteint trois mois en moyenne, et jusqu'à neuf mois. Ces résultats 
confirment ainsi la recommandation de supprimer tous les vieux cacaoyers entre la $3^{\text {ème }}$ et la $4^{\text {ème }}$ année après la mise en place (Petithuguenin et al., 2004), pour, d'une part, favoriser la production, et, d'autre part, éviter de fortes attaques des insectes et des maladies déjà présents sur ces vieux cacaoyers. Il est donc clairement mis en évidence ici l'intérêt d'une bonne gestion de l'ombrage des vieux cacaoyers au cours de la phase d'établissement des jeunes plants de cacaoyers.

$\mathrm{Au}$ regard de l'ensemble des résultats obtenus, la replantation sous vieux cacaoyers s'est avérée plus efficace qu'après les antécédents jachères de courtes durée. Cependant, cette technique ne peut être utilisée pour la rénovation des vergers dégradés par la maladie du swollen shoot. En effet, les vieux cacaoyers malades constituent la principale source d'inoculum du virus qui favorise l'infection rapide des jeunes cacaoyers replantés dès la deuxième année (Dzahini-Obiatey et al., 2006). Aussi, la technique de replantation recommandée dans les zones infectées par le swollen shoot consiste-t-elle à arracher totalement les cacaoyers malades, ainsi que leurs voisins immédiats, et à protéger la parcelle replantée par une barrière d'au moins $10 \mathrm{~m}$ de large. Cette barrière doit être constituée par une culture non hôte du virus du swollen shoot (Dzahini-Obiatey et al., 2006).

\section{REMERCIEMENTS}

Les auteurs renouvellent leurs remerciements au programme de développement durable des cultures pérennes en Côte d'Ivoire (STCP) qui a entièrement financé la réalisation des études dont les résultats de la composante sur la replantation sont résumés dans le présent article.

\section{RÉFÉRENCES BIBLIOGRAPHIQUES}

Aguilar, P. et Jadin, P. 1997. La replantation des vieilles cacaoyères en Afrique. Afrique agriculture 246 : 66-67.

Anonyme. 1999. L'agriculture ivoirienne à l'aube du $21^{\text {ème }}$ siècle. Éditions Dialogue Production - Multimédia, Abidjan, Côte d'Ivoire. 295p. http://www.hubrural.org/IMG/pdf/ cote ivoire agriculture 21e siecle.pdf. Accédé le 02 Novembre 2010.

Anonyme. 2003. Filière Café-Cacao : quelles stratégies face aux nouveaux enjeux et défis ? Actes des Journées portes ouvertes 2003 du Café et du Cacao, du 9 au 11 juillet 2003, Abidjan, Côte d'Ivoire. 12p.

Assiri, A.A. 2007. Identification des pratiques paysannes dans la conduite des vergers de cacaoyers en Côte d'Ivoire. Mémoire de Diplôme d'Études Approfondies (DEA), UFR STRM, Université de Cocody-Abidjan, Côte d'Ivoire. 56p.

Assiri, A.A., Yoro, G.R., Deheuvels, O., Kébé, B.I., Kéli, Z.J., Adiko, A. et Assa, A. 2009. Les caractéristiques agronomiques des vergers de cacaoyer (Theobroma cacao L.) en Côte d'Ivoire. Journal of Animal \& Plant Sciences 2(1): 55-66.

Assiri, A.A. 2010. Etude de la régénération cacaoyère en côte d'ivoire : impact des techniques de réhabilitation et de replantation sur le développement et la productivité des vergers de cacaoyers (Theobroma cacao L.) en relation avec l'état du sol. Thèse de Doctorat Unique de l'UFR STRM, Université de Cocody-Abidjan, Côte d'Ivoire. 170p.

Assiri, A.A., Kacou, E.A., Assi, F.A., Ekra, K.S., Dji, K.F., Couloud, J.Y. et Yapo, A.R. 2012. Rentabilité économique des techniques de réhabilitation et de replantation des vieux vergers de cacaoyers (Theobroma cacao L.) en Côte d'Ivoire. Journal of Animal \& Plant Sciences 14 (2): 1939-1951.

Bertrand, B. et Jadin, P. 1992. Recherche d'un itinéraire technique pour la replantation des cacaoyères au Togo : étude comparative de divers modes de plantation des cacaoyères. Café Cacao Thé 36 (2) : 115-120.

Besse, J. 1972. Comparaison de deux méthodes d'établissement de cacaoyère. Café Cacao Thé 26 (4) :317-332.

Brou, Y. T., N'Goran, J.A.K., Bicot, S. et Servat, E. 2003. Risque climatique et production agricole en Côte d'Ivoire : effet des variations pluviométriques sur la production cacaoyère. Actes de la $14^{\text {ème }}$ Conférence internationale sur la recherche cacaoyère, du 18 au 23 octobre 2003, Accra, Ghana : 259-267. 
Brou, Y.T., Francis, A. et Bigot, S. 2005. La variabilité climatique en Côte d'Ivoire : entre perceptions sociales et réponses agricoles. Cahiers d'études et de recherches francophones 14 (6) : 533-540.

Brou, Y.T. 2005. Climat, mutations socioéconomiques et paysages en Côte d'Ivoire. Mémoire de synthèse des activités scientifiques présenté en vue de l'obtention de l'Habilitation à Diriger des Recherches, Université des Sciences et Technologies de Lille, France. 212p.

Clough, Y., Faust, H. and Tscharntke, T. 2009. Cacao boom and bust: sustainability of agroforests and opportunities for biodiversity conservation. Conservation Letters 2: $197-$ 205.

Dzahini-Obiatey, H., Akumfi, A.G. and Ollennu, L.A. 2006. Control of cocoa swollen shoot disease by eradicating infected trees in Ghana: A survey of treated and replanted areas. Crop Protection 25: 647-652.

FAO. 2003. L'étude prospective du secteur forestier en Afrique (FOSA) : Document national de prospective - Côte d'Ivoire. http:/ /www.fao.org/docrep/003/X6780F/ X6780F00.htm. Accédé le 11 novembre 2008.

FAO. 2009. Harmonized world soil database (HWSD). FAO (Food and Agriculture Organization), Rome, Italy. 38p.

FAO. 2006. World reference base for soil resources 2006: a framework for international classification, correlation and communication. World soil resources reports, $\mathrm{N}^{\circ} 103,2^{\text {nd }}$ edition. FAO (Food and Agriculture Organization), Rome, Italy. 145p.

Freud, E.H., Petithuguenin, P. et Richard, J. 2000. Les champs de cacao : un défi de compétitivité Afrique-Asie. Éditions Karthala et CIRAD, Collection Économie et développement, Paris, France. 207p.

Gnahoua, G.M. 2004. Contribution des légumineuses à la régénération des jachères : intérêts et limites des arbres fixateurs d'azote en zone forestière de Côte d'Ivoire. Thèse de Docteur-Ingénieur de l'UFR STRM, Université de Cocody - Abidjan, Côte d'Ivoire. $132 \mathrm{p}$.

ICCO. 2015. Quarterly Bulletin of Cocoa Statistics, Vol. XLI, No. 2, Cocoa year 2014/15.
International Cocoa Organization (ICCO), Westgate House, London, United Kingdom. http://www.icco.org/statistics/otherstatistical-data.html. Accessed 9 September 2015.

Jarrige, F. et Ruf, F. 1990. Comprendre la crise du cacao. Café Cacao Thé 34 (3) : 213-222.

Kassin, K.E., Doffangui, K., Yoro, G.R. et Assa, A. 2008. Variabilité pluviométrique et perspectives pour la replantation cacaoyère dans le Centre Ouest de la Côte d'Ivoire. Journal of Applied Biosciences 12 : 633- 641.

Kassin, K.E. 2009. Études des conditions pédoclimatiques pour la replantation cacaoyère au Centre-Ouest de la Côte d'Ivoire : cas des départements de Divo et Gagnoa. Thèse de Doctorat unique de l'UFR STRM, Université de Cocody - Abidjan, Côte d'Ivoire. $112 \mathrm{p}$.

Kébé, B.I., N'Guessan, K.F., Tahi, G.M., Assiri, A.A., Koko, L.K., Kohi, N.J., Irié, B.Z. et Koffi, N. 2009. Bien cultiver le cacaoyer en Côte d'Ivoire. Fiche Technique. Centre National de Recherche Agronomique (CNRA) - CTA. 4p. ht tp://www.cnra.ci/downloads/ cultiver cacao ci.pdf. Accédé le 02 Novembre 2010.

Lachenaud, Ph. 1987. L'association cacaoyerbananier plantain : étude de dispositifs. Café Cacao Thé 31 (3) : 195-201.

Léonard, E. et Oswald, M. 1996. Une agriculture forestière sans forêt : changements agroécologiques et innovations paysannes en Côte d'Ivoire. Natures - Sciences - Sociétés 4 (3) : 202-216.

Losch, B. 1994. Cacaos africains : comment la Côte d'Ivoire a devancé le Cameroun. Plantations, Recherche, Développement 1 (2) : 15-27.

Naï Naï, S., Cheyns, E. et Ruf, F. 2000. Adoption du palmier à huile en Côte d'Ivoire. Journal français des Oléagineux, Corps Gras, Lipides 7 (2) : 155-165.

Oppong, F.K., Asante, E.G., Osei-Bonsu, K., Acheampong, K. and Amoah, F.M. 2003. Agronomic and economic evaluation of different weed management options in young cocoa. Actes de la $14^{\text {ème }}$ Conférence internationale sur la recherche cacaoyère, du 18 au 23 octobre 2003, Accra, Ghana : 291301. 
Oppong, F.K., Opoku-Ameyaw, K., OforiFrimpong, K. and Owusu-Ansah, F. 2006. Investigation into the period of weed competition in young Theobroma cacao $\mathrm{L}$. Actes de la $15^{\text {ème }}$ Conférence internationale sur la recherche cacaoyère, du 9 au 10 octobre 2006, San José, Costa Rica: pp. 281290.

Petithuguenin, P. 1995. Cacaoculture et évolution du milieu : une contribution à la réflexion sur la reproductibilité de ces systèmes de cultures. Actes du séminaire sur la Fertilité $d u$ milieu et stratégies paysannes sous les tropiques humides, du 13 au 17 novembre 1995, Montpellier, France : pp. 340-349.

Petithuguenin, P., Deheuvels, O. and Assiri, A.A. 2004. Sustaining cocoa cultivation. In: Cocoa futures: a source book of some important issues facing the cocoa industry. Flood, J. and Murphy, R. (Éd.), CABI-FEDRECAFÉ, USDA, Chinchina, Colombie: pp. 128-140.

Ruf, F. 1995. Booms et crises du cacao : les vertiges de l'or brun. Éditions Karthala \& CIRAD, Collection Économie et développement, Paris, France. $459 \mathrm{p}$.

Ruf, F. 2000. Déterminants sociaux et économiques de la replantation. Journal français des Oléagineux, Corps Gras, Lipides 7 (2) : 189196.
Ruf, F. et Allangba, K. 2001. Les difficultés de la replantation. Quel avenir pour le cacao en Côte d'Ivoire. Actes de la Conférence internationale sur l'avenir des cultures pérennes : Investissement et durabilité en zones tropicales humides, du 5 au 9 juin 2001, Yamoussoukro, Côte d'Ivoire. 13p.

Shenk, M.D. 1971. Weed control in cocoa. World farming 12 (10): 12-13.

Snoeck, D. 2006. The soil diagnostic method for formulating fertilizer requirements on cocoa in Ghana. Actes de la $15^{\text {ème }}$ conférence internationale sur la recherche cacaoyère, du 9 au 10 octobre 2006, San José, Costa Rica: pp. 387-394.

Vaast, Ph. 1987. Présentation des systèmes de régénération cacaoyère en milieu paysan au Togo. Actes de la $10^{\text {ème }}$ conférence internationale sur la recherche cacaoyère, du 17 au 23 mai 1987, Saint Domingue, République Dominicaine : pp. 63-69.

Van Reeuwijk, L.P. 2006. Procedures for soil analysis. $7^{\text {th }}$ edition. ISRIC Technical paper 9, Wageningen, the Netherland. 101p. http:// www.isric.org/ISRIC/webdocs/docs/ tp9blanco.pdf. Accessed 14 February 2008.

Wood, G.A.R. and Lass, R.A. 1986. Cocoa. Éditions Longman Scientific and Technical Agriculture Series, Fourth edition, Londres, Angleterre. 620p. 